\title{
Liberia and Sierra Leone could see 1.4 million Ebola cases by January
}

\author{
Michael McCarthy
}

Seattle

Without additional intervention the number of cases of Ebola virus disease in the west African countries of Liberia and Sierra Leone could reach 1.4 million by the end of January, a report by the US Centers for Disease Control and Prevention (CDC) has found. ${ }^{1}$

"If conditions continue without scale-up of interventions, cases will continue to double approximately every 20 days, and the number of cases in West Africa will rapidly reach extraordinary levels," the report said.

However, the report's findings also indicated that the epidemic could be quickly brought under control by rapidly increasing the number of patients being treated in medical care facilities and by improving home care and prevention in affected communities.

The CDC's estimate is the second dire prediction to come out this week. A study by the World Health Organization and Imperial College London, published in the New England Journal of Medicine, projected that the number of Ebola cases will rise "exponentially" to more than 20000 by the beginning of November. This was significantly higher than previous WHO estimates and raised the possibility that the disease might become endemic in west Africa.

The Ebola virus is spread mainly through contact with bodily fluids from a symptomatic patient. Transmission can be prevented by isolation of patients, rigorous infection control, and the adoption of safe burial practices.

The first cases of Ebola virus disease in the current epidemic were reported in March 2014. According to the figures reported in the New England Journal of Medicine study, as at 14 September 2014 there have been a total of 4507 probable and confirmed cases, including 2296 deaths from Ebola in five countries in west Africa-Guinea, Liberia, Nigeria, Senegal, and Sierra Leone. The overall case fatality rate was estimated to be $70.8 \%$.

In the new CDC study, researchers created a modeling tool, called EbolaResponse, to project the course of the Ebola epidemic in Liberia and Sierra Leone under various scenarios. These two countries were chosen because the available data were the best fit for the model.

The researchers reported that in the worst case scenario-in which there is no substantial rise in the number of patients receiving care in medical facilities nor effective implementation of home care and community prevention practices that reduce the risk of transmission, such as the adoption of safe burial practices - the total number of cases in both countries would double every 20 days, reaching 21000 by the end of September and 1.4 million by 20 January 2015 .

On the other hand, if by late December $70 \%$ of patients were placed either in a medical facility or in a setting where the risk of transmission was reduced the epidemic could be almost ended by 20 January 2015 . "Once $70 \%$ of patients are effectively isolated, the outbreak decreases at a rate nearly equal to the initial rate of increase," the report said.

However, the model indicated that there is substantial cost associated with delaying implementation of the recommended interventions. Assuming that it will take 90 days to set up enough treatment units to provide care for $70 \%$ of patients with Ebola, the model predicts that every 30 day delay in starting the process will result in a tripling of the number of daily cases that will occur at the peak of the epidemic.

For example, if the ramp up of the response to the epidemic began on the day the report was published, 23 September, and reached the $70 \%$ coverage goal by the end of December, daily cases would be projected to peak at 3408 and then fall to less than 300 daily cases by 20 January.

If, however, the implementation initiative were delayed by 30 days, a peak of 10646 daily cases would be expected. If implementation was delayed another 30 days, a peak of 25847 cases would be expected by 20 January, the last date included in the model.

In a telephone briefing for reporters, CDC director Tom Frieden said the model's projections were for the most part encouraging. "The bottom line here is that a surge now can break the back of the epidemic. It also shows that there are severe costs of delay," he said.

Frieden said he was confident the model's most "dire projections are not going to come to pass" because of an increasingly effective local and international response to the epidemic. "If we move fast enough, we can turn it around," he said.

To help local health officials and international agencies in their planning, the CDC has made the modeling tool used in the study available online at http://stacks.cdc.gov/view/cdc/24900.

1 Meltzer MI, Atkins CY, Santibanez S, Knust B, Petersen BW, Ervin ED, et al. Estimating the future number of cases in the Ebola epidemic-Liberia and Sierra Leone, 2014-2015. Morb Mortal Wkly Rep 2014:63:1-14.

2 WHO Ebola Response Team. Ebola virus disease in west Africa: the first 9 months of the epidemic and forward projections. N Engl J Med [Epub ahead of print]. 
